\title{
On the graphitization of diamond surfaces: the importance of twins
}

\author{
G. Jungnickel $^{\text {a }}$, C.D. Latham ${ }^{\text {a }}$, M.I. Heggie ${ }^{\text {a }}$, Th. Frauenheim ${ }^{\text {b** }}{ }^{*}$ \\ a Department of Computer Science, University of Exeter, Prince of Wales Road, Exeter EX4 4PT, U.K., \\ a Institut für Physik, Theoretische Physik III, Technische Universität, D-01079 Chemnitz, Germany \\ Received 11 August 1995; accepted in final form 21 September 1995
}

\begin{abstract}
$A b$ initio total energy calculations reported recently (M.I. Heggie, C.D. Latham, R. Jones and P.R. Briddon, Phys. Rev. B, 50 (1994) 5937) revealed that the tetrahedrally bonded icosahedral $\mathrm{C}_{100}$ molecule spontaneously decomposed into two concentric fullerenes $\left(\mathrm{C}_{20}\right.$ and $\left.\mathrm{C}_{80}\right)$. This $\mathrm{C}_{100}$ molecule belongs to a series of structures that may be viewed as the diamond analogues of fullerenes (L. Zeger and E. Kaxiras, Phys. Rev. Lett., 70 (1993) 2920). Since these molecules can be seen to be effectively a heavily twinned molecular diamond their stability is important in the context of investigating the diamond $\{111\}$ surface where a twin emerges. We present ab initio self-consistent calculations on a rather small $\mathrm{C}_{40} \mathrm{H}_{36}$ molecule representing the core of a twin intersecting two diamond $\{111\}$ surfaces and compare the results with that obtained with a non-selfconsistent density-functional based tight-binding method. Since the latter is also capable of handling larger and periodic models in a molecular dynamics relaxation we use it to study the graphitization effect in dependence of temperature. We find nearly the same ground state for the small molecule which is clearly due to a graphitization and find strong surface graphitization for a model of 128 carbon atoms at elevated temperatures. At $2700 \mathrm{~K}$ the top layer of this periodic model completely delaminates.
\end{abstract}

Keywords: Molecular dynamics; Diamond surface; Twins; Graphitization

\section{Introduction}

Recent $a b$ initio total energy calculations [1] revealed that the $\mathrm{C}_{100}$ molecule, which belongs to a series of structures that may be viewed as the diamond analogues of fullerenes [2], decomposes into two concentric fullerenes $\mathrm{C}_{80}$ and $\mathrm{C}_{20}$. We have carried out $\mathrm{ab}$ initio self-consistent calculations on the $\mathrm{C}_{40} \mathrm{H}_{36}$ molecule and use the results to study the graphitization of diamond surfaces.

The graphitization of diamond surfaces and the tetrahedrally bonded icosahedral $\mathrm{C}_{100}$ molecule $[1,2]$ is attracting attention because there is substantial experimental evidence that graphitic precursors in the very early stages of diamond depostion occur and promote diamond nucleation, see e.g. $[3,4]$. This is supported by the recent EELS investigation by Fallon and Brown where amorphous carbon layers mostly graphitic in character were found in grain boundaries [5] and by the Raman and HRTEM observations by Kang et al. [6] who find amorphous films growing first on silicon substrates prior to diamond deposition. Other experimental evidence is discussed in our recent work [7], which includes circumstances where diamond becomes graphitised or amorphous such as, under ion implantation with non-reactive ions $[8,9]$, the result of heat treatment [10], or in measurements of friction coefficients by sliding diamond against diamond [11]. Another important discovery is that ultradispersed diamond starts to graphitize from the surface gradually inwards with an onset temperature of about $1200 \mathrm{~K}$ [12]. Related to these observations is that heteroepitaxially oriented nucleation of diamond particles on graphite prism planes can occur [13]. This led to the hypothesis by Ref. [4] that diamond nucleation begins by hydrogenation of graphitic edges formed at the first stage of deposition, then subsequent hydrogen abstraction and carbon radical insertion reactions convert this material into epitaxially oriented diamond nuclei.

Nucleation and growth mechanisms have been studied by a wide variety of theoretical methods. These have been extensively reviewed in Ref. [14]. Earlier work tended to concentrate on gas phase and surface reactions occuring in diamond CVD [15-21], and it is only recently that detailed atomistic modelling of graphitization by semiempirical $[22,23]$ and parameter-free [24,25] methods has been done. One observation is that while flat $\{111\}$ surfaces show only a flattening of the surface which cannot be considered to be a true graphitization $[7,23]$, any event that pins 
the surface down at some point (e.g. at surface steps [22], near flakes [23], or in the vicinity of bond rearrangements typical for the Pandey $\pi$-bonded chain reconstruction $[7,25]$ ) causes a more pronounced even spontaneous [22] graphitization. Another interesting aspect was pointed out recently by Heggie et al. [24] who found the neighbourhood of a twin intersecting two $\{111\}$ surfaces graphitizes spontaneously too, and discussed the importance of surface curvature due to the twin in motivating the reaction. A potential weakness of the twin model was it used a rather small finite cluster where all the atoms were allowed to relax, so the behaviour of the surrounding bulk crystal was poorly described.

In the following we report on new studies of the latter calculations including fixed boundary selfconsistent field (SCF) calculations and calculations done with the density-functional based tight-binding (DF-TB) method [26] for the small molecule representing the twin and molecular dynamics studies using this DF-TB scheme on a periodic slab model containing 128 carbon atoms.

The paper is organized as follows. In Section 2 we briefly summarize the computational methods used throughout the paper. Section 3 presents the results obtained for the finite cluster calculations, whereas in Section 4 we present the observations for the periodic models relaxed by constant temperature molecular dynamics.

\section{Computational methods}

\subsection{Ab Initio Self-consistent field method}

This method gives the total energy of atomic clusters by solving self-consistently the Kohn-Sham equations obtained from the local density approximation (LDA) [27-30]. The Ceperly-Alder expression, parameterised by Perdew and Zunger, is used to calculate the exchange and correlation energy term [31,32]. The wavefunction and charge density of atoms are described by a real-space basis set of Gaussian-type functions centred on the cartesian coordinates of the nuclei, and, optionally at bond-centred sites. Both $s$ and $p$-type fitting functions are used for the wavefunction part of the basis, while the charge density uses only $s$-type functions. The number of fitting functions centred on each atom is chosen so that there are more on those near to critical regions and fewer on atoms occupying peripheral positions. The norm-conserving pseudopotentials of Bachelet et al. are used in the calculation, except for hydrogen atoms which have a simple Coulomb potentential [33]. Forces between atoms are given by an analytical formula derived from the total energy expression.

\subsection{Density-functional based tight-binding method}

Within this method which was previously outlined as a density-functional based tight-binding (DF-TB) approximation $[26,34]$ the interatomic forces are calculated numerically using a tight-binding expression for the total energy of the system

$$
E_{t o t}=\sum_{i}^{o c c .} \varepsilon_{i}+\sum_{\mathbf{R}, \mathbf{R}^{\prime}} \Phi\left(\left|\mathbf{R}-\mathbf{R}^{\prime}\right|\right) .
$$

$\sum_{i}^{o c c .} \varepsilon_{i}$ is the sum of occupied Kohn-Sham eigenvalues based on local density functional theory and is referred to as the band structure energy. $\Phi$ is a universal short-range repulsive pair-potential that is adjusted to be the difference of the band structure energy and the cohesive energy as calculated in a self-consistent calculation for a diatomic carbon molecule versus distance. Additionally, the pair potential is chosen to obtain the correct lattice constants, bulk moduli, and cohesive energies of diamond and graphite.

Within a two-center approach the band structure energy is evaluated by solving the Kohn-Sham equation within a non-self-consistent treatment. All necessary Hamiltonian and Overlap matrix elements are calculated within density-functional theory using the linear combination of atomic orbitals (LCAO)-LDA approach rather than fitted to crystalline data as in empirically parametrized TB-methods. For this, we expand the wave-function of the many-atom structure into a minimal basis of self-consistently derived "compacted" valence electron orbitals of free atoms [26], which are represented as Slater-type functions. Since the parametrization of all matrix elements vs. distance is well practised, in guaranteeing sufficient accuracy combined with high transferability the method becomes as simple and efficient as empirical nonorthogonal tight-binding schemes and thus is useful for molecular dynamics (MD) simulations.

\section{Finite cluster conjugate gradient simulations}

Recent $a b$ initio studies [24] of a finite model for a twin emerging at a diamond surface showed a spontaneous graphitization of this model surface (see Fig. 1). The model contained 40 carbon atoms. The back surfaces in this model was saturated with 36 hydrogen atoms in order to simulate an extended structure. The (111) facets were left bare. Periodicity in the crystal directions perpendicular to the surface could not be exploited. The atoms belonging to the cluster were all allowed to relax freely; the bottom carbon atoms and the attached hydrogens that should have simulated the stiffness of the bulk could, therefore, move significantly. From the inital cluster showing the typical diamond structure with a twin, see Fig. 1(a) for which the 
carbon-carbon bond lengths was set to $1.54 \AA$ a second one was derived by modifying the coordinates of the surface atoms anticipating a delamination of the top layer as shown in Fig. 1(b). Both these clusters were then relaxed within the self-consistent field method by a conjugate gradient method in order to find the ground state of the clusters.

As is pointed out in Ref. [24] the relaxation starting from the diamond structure (a) caused serious numerical problems when trying to reach selfconsistency because of the number of dangling bonds at the surface that give rise to electron levels at the Fermi level of this particular molecule. Therefore, to avoid unstable calculations the eigenstates were filled according to a Fermi distribution with a finite temperature of $0.03 \mathrm{eV}$. This treatment was switched off after the relaxation converged to ensure that the ground state off the system is not affected by this redistribution of the occupation of electron levels. It was not applied to structure (b).

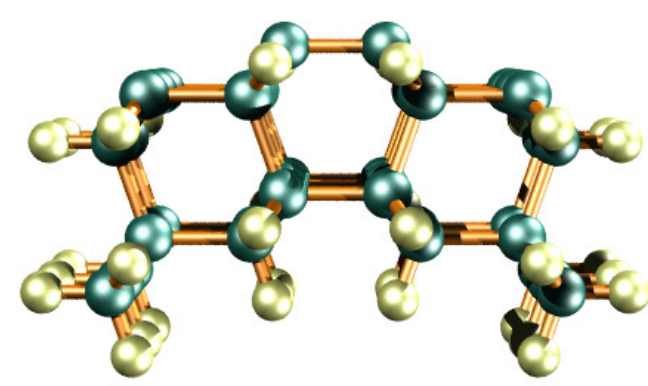

(a)

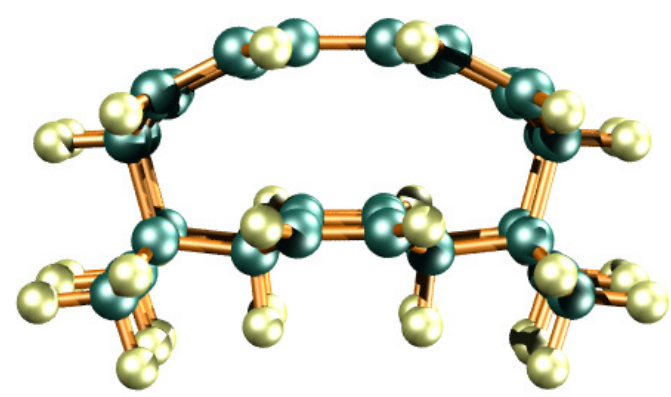

(b)

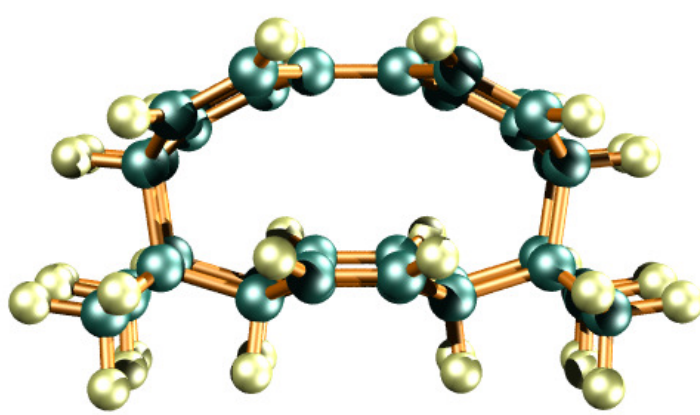

(c)

Figure 1: The $\mathrm{C}_{40} \mathrm{H}_{36}$ model used in the cluster studies. Conjugate gradient relaxation of the initial diamond structure (a) and a modified structure anticipating graphitization (b) generates very much the same final state (c) during the SCF calculations.

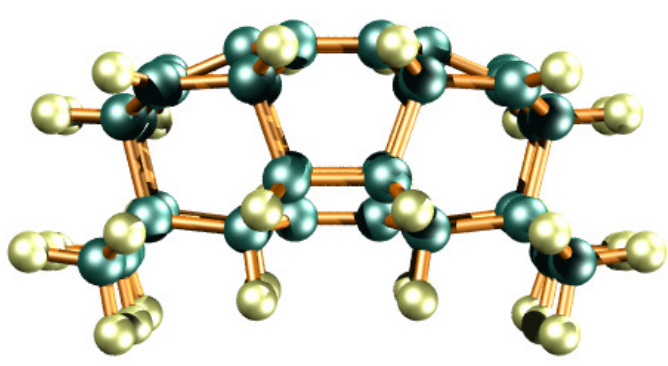

(a)

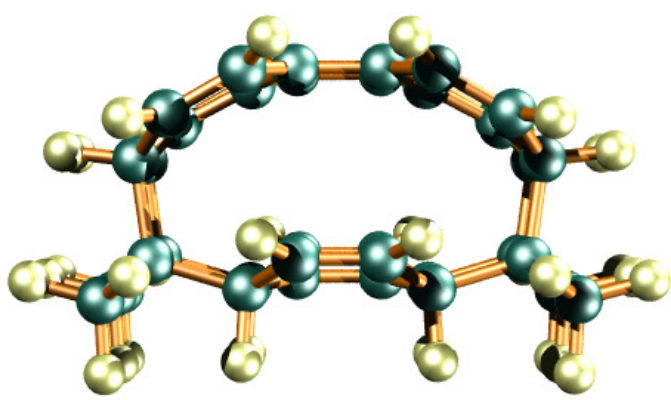

(b)

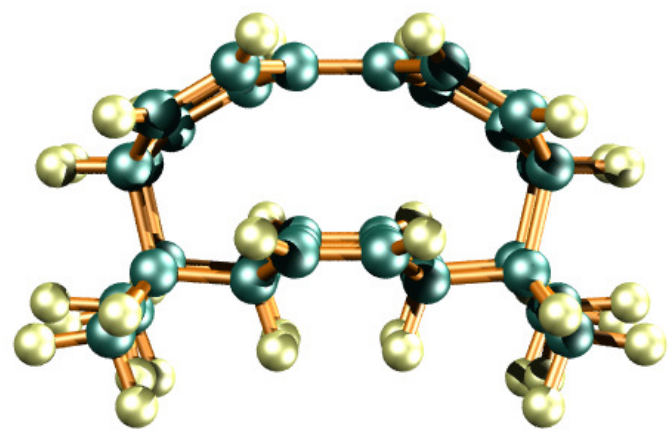

(c)

Figure 2: The final models after conjugate gradient relaxation using the DF-TB method starting from the initial diamond structure (a) and the modified one (b) are different, since the former is a metastable state. Using the SCF method and fixing some of the attached hydrogen atoms the final relaxation result is the model shown in (c).

The final result after relaxing both the initial and the modified structure was found to be almost indistinguishable and is clearly a graphitized structure as may be seen in figure 1(c). The decrease in total energy was calculated to be $28.5 \mathrm{eV}$ starting from the initial model (a) and $2.7 \mathrm{eV}$ starting from the modified version (b) which is already $25.8 \mathrm{eV}$ lower in energy than (a).

To check the applicability of the simpler tightbinding procedure, we have re-calculated the problem and the results are given in figure 2(a) and (b). First of all, we find the graphitized structure that is the broken bond structure presented in figure 2(b) being the ground state with this method, too. This is found when starting with a conjugate gradient relaxation from model 1(b). It should be noticed that the ground state molecule in the DF-TB as well as in the SCF method consists of $22 s p^{2}$-like atoms and $18 s p^{3}$ like atoms and no mixed hybridization state when applying an analysis recently introduced for studies of amorphous carbon [35]. This analysis is based on the 
requirement that a three-fold coordinated carbon atom is in an $s p^{2}$-like state only if it would generate a minimum in the local density of states due to a strong $\pi$ interaction with its $\pi$-bonded neighbours. Moreover, the structural characteristics of the ground state models as given in table 1 are fairly equal in both cases. The average bond length between the $s p^{2}$-like atoms is $\approx 1.40 \AA$ slightly smaller as the typical value for an extended graphite crystal.

However, starting from the initial diamond structure 1(a) either due to non-selfconsistency or the particular step width in the conjugate gradient algorithm used the tight-binding approximation captured a metastable state with the inner bonds broken and the outer ones still in the original position. From that we expect the method to be suitable to find graphitized structures if they are favoured because of the change in total energy which in the case studied here is $21.4 \mathrm{eV}$ relative to the initial diamond structure and $3.7 \mathrm{eV}$ relative to the modified starting structure. Under certain conditions, however, we have to check the result of a simple conjugate gradient relaxation for not being trapped into local potential minima. This problem is less important when the size of the molecule gets larger and molecular dynamics is used as the relaxation tool.

A new aspect is that we have repeated with constraints the self-consistent calculation for the model in figure 1(a) by fixing the four hydrogen atoms below the twin core and another four at the corners of the model. The objective of this is to ensure that the original conclusion that the surface intersected by a twin graphitizes spontaneously [24] is still valid even if the cluster is constrained. The result is shown in figure 2(c) and is only slightly different from the previous result in that the top layer is now shifted outwards a little more. The change in total energy has been calculated to be $25.6 \mathrm{eV}$. As can be seen from table 1 there is a strain on both the $s p^{3}-s p^{3}$ and the $s p^{2}-s p^{2}$ bonds, making their average lengths larger and the average bond angle at three-fold coordinated atoms slightly smaller.

We have shown that both the unconstrained and the constrained back surface the clusters in the SCF calculation reconstruct spontaneously to a graphitic-like form. Taking this as as a benchmark for the less rigor- ous tight-binding calculation, shows that this method is able to reproduce very nearly the same ground state properties as the more sopisticated technique.

\section{Molecular dynamics studies within the DF-TB scheme}

In order to obtain a more realistic description of the twin intersecting the two diamond $\{111\}$ facets we then used models with 128 carbon atoms the bottom of which was saturated by 16 hydrogen atoms that were held fixed to simulate the bulk stiffness. These models were periodic but include a large vacuum region in the direction perpendicular to the surface. Moreover, due to the periodic boundary condition these models contain not only one twin leading a convex surface form but a second one with a concave surface. This arrangement is usually called a microtwin.

First, we applied a conjugate gradient algorithm to investigate the structure at $0 \mathrm{~K}$. Secondly, we used a standard Verlet molecular dynamics algorithm to simulate the behaviour of the model at finite constant temperature of $1200 \mathrm{~K}$ and $2700 \mathrm{~K}$. The resulting structures are shown in Fig. 3(a), (b), and (c). Fig. 3(a) is the final state of the conjugate gradient relaxation showing a less pronounced tendency towards spontaneous graphitization than reported recently [24]. The top double layer is only very slightly shifted outwards. The lengths of the bonds between the top and the second double layer are stretched to $109 \%$ of the diamond value. There is no graphitization of the surface as is shown in figures 1(c) or 2(b) and (c). This observation is consistent with the result obtained on a flat bare $\{111\}$ surface [23], where no spontaneous graphitization was found for models with 216 atoms. One might argue that the DF-TB method again is in a local minimum of the potential, but in turn it could be, that the result for a larger periodic model including a microtwin is indeed somewhat different from the cluster calculations as was already mentioned in Ref. [24].

We then investigated the structure by molecular dynamics at temperatures of $1200 \mathrm{~K}$ and $2700 \mathrm{~K}$. Fig. 3(b) and 3(c) show the final models of these calculations after equilibrating the obtained structures in a simulated annealing run for a total time of $1.2 \mathrm{ps}$ at the considered temperature. As in recent studies of

Table 1: Comparison of ground state properties for the different calculation techniques. When fixing some hydrogen atoms at the back surface, the carbon-carbon bonds are strained.

\begin{tabular}{|c|c|c|c|c|c|c|}
\hline \multirow[t]{2}{*}{ Model } & \multicolumn{3}{|c|}{ Average bond length $(\AA)$} & \multicolumn{2}{|c|}{ Average bond angle (deg.) } & \multirow{2}{*}{$\begin{array}{l}\text { Average distance }(\AA) \\
\text { between atoms at the core } \\
\text { (former bonds) }\end{array}$} \\
\hline & $s p^{2}-s p^{2}$ & $s p^{2}-s p^{3}$ & $s p^{3}-s p^{3}$ & $s p^{2}$ sites & $s p^{3}$ sites & \\
\hline SCF (unconstrained optimization) & $1.406 \pm 0.059$ & $1.516 \pm 0.012$ & $1.555 \pm 0.021$ & $120.0 \pm 2.3$ & $111.6 \pm 3.6$ & 3.11 \\
\hline DF-TB & $1.392 \pm 0.056$ & $1.503 \pm 0.009$ & $1.547 \pm 0.020$ & $120.0 \pm 2.0$ & $111.2 \pm 3.1$ & 3.02 \\
\hline SCF (eight H-atoms held fixed) & $1.415 \pm 0.060$ & $1.512 \pm 0.005$ & $1.558 \pm 0.049$ & $119.4 \pm 3.4$ & $111.6 \pm 4.0$ & 2.81 \\
\hline
\end{tabular}


plain hydrogen free diamond $\{111\}$ surfaces at constant temperature [23] we obtain a clear tendency towards graphitization of the surface. In contrast, here, the top layer delaminates totally at $2700 \mathrm{~K}$. The minimum distance of the delaminated layer to the next lower lying atoms is about $3.0 \AA$ whereas the average distance of the top double layer from the second one in the case of the flat bare surfaces was found to be only about $2.4 \AA$ [7]. In agreement with the previous studies at $1200 \mathrm{~K}$ the surface at the (111) facets is not completely detached from the next double layer and is still preserving strained $\sigma$ bonds. However, it is important to note that a strong graphitization effect starts in the neighbourhood of the convex twin which is also the case for subsequent graphitization steps in the next layers when the surface is completely detached at $2700 \mathrm{~K}$. The same effect cannot be discovered for the concave twin which seems to provide an anchoring point for the top layer similar to those discussed in the introduction. Another difference from the previous studies is that in them the surface energy for the flat surfaces increased linearly with temperature $[7,23]$ whereas here the surface energy of the model after the MD run at $2700 \mathrm{~K}$ is lower than the surface energy at $1200 \mathrm{~K}$ by about $5.7 \mathrm{~J} \mathrm{~m}^{-2}$ owing to the graphitization.

\section{Conclusions}

We have found the graphitized structure to be the ground state for a model containing 40 carbon and 36 hydrogen atoms simulating the core of a twin intersecting two diamond (111) facets within a sophisticated self-consistent field method as well as in a simpler density-functional based tight-binding scheme.

We then applied the DF-TB method to molecular dynamics simulation of a larger periodic model of a twin intersecting a diamond surface and found a strong graphitization starting at about $1200 \mathrm{~K}$ especially in the neighbourhood of the twin making a convex surface. In contrast, the second twin introduced in this model because of the periodic boundary condition making a concave surface form is rather an anchoring point for the top layer. At $2700 \mathrm{~K}$ we observe a total delamination of the top layer and a decrease in the internal energy of the system showing a true graphitization happening.

The occurence of graphitic structures at growth temperature suggests that such layers may be involved not only in the nucleation steps but also in growth processes as conversion layers that are converted to diamond by the hydrogenation conversion mechanism suggested in Ref. [4]. A more extended discussion of this point and a review of several graphitization effects found will be given elsewhere [7].

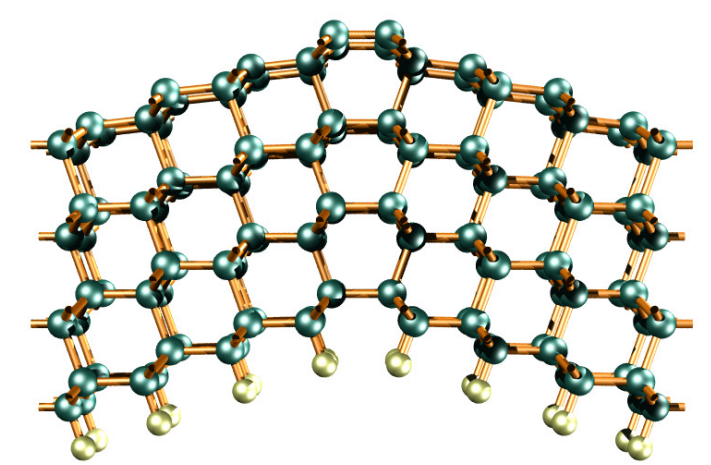

(a)

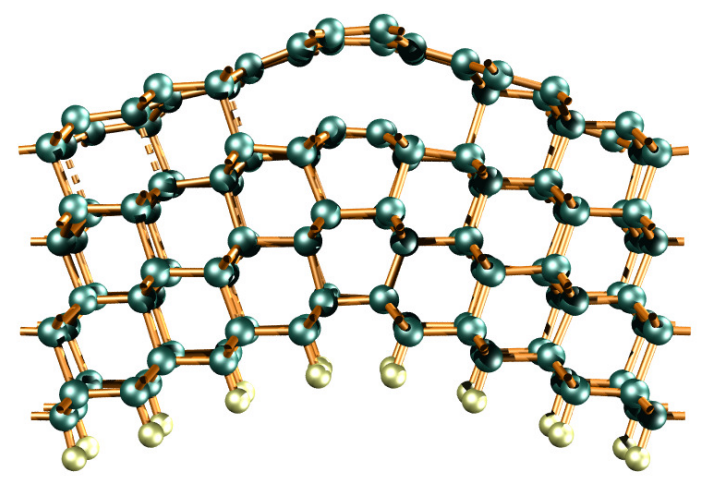

(b)

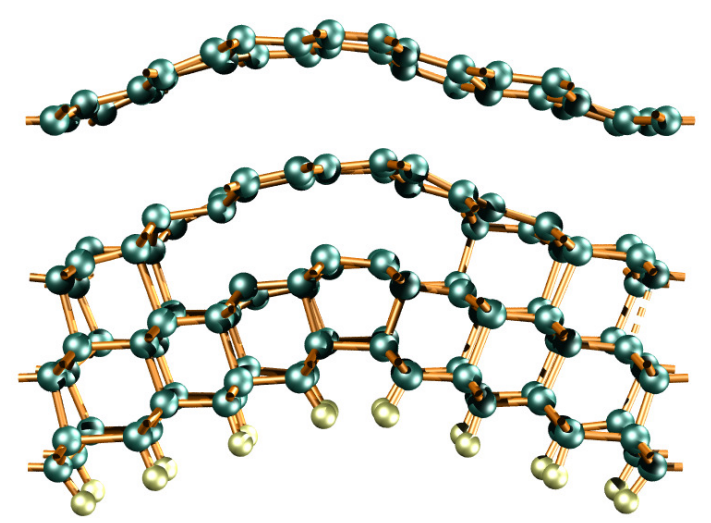

(c)

Figure 3: (a) The periodic $\mathrm{C}_{128} \mathrm{H}_{16}$ model at $0 \mathrm{~K}$ of a twin intersecting a surface shows no spontaneous graphitization. (b) After MD for $1.2 \mathrm{ps}$ at $1200 \mathrm{~K}$ graphitization has clearly started at the convex twin. (c) After MD for $1.2 \mathrm{ps}$ at $2700 \mathrm{~K}$ the top layers are totally delaminated and sublayer graphitization is starting at the twin.

\section{Acknowledgements}

We greatfully acknowledge support by the Deutsche Forschungsgemeinschaft (DFG) and the EPSRC of Great Britain.

\section{References}

[1] M. I. Heggie, C. D. Latham, R. Jones, and P. R. Briddon, Physical Review B 50 (1994) 5937.

[2] L. Zeger and E. Kaxiras, Phys. Rev. Lett. 70 (1993) 2920.; L. Zeger and E. Kaxiras, Comp. Mat. Sci. 1 (1993) 403.

[3] J.C. Angus, A. Argoitia, R. Gat, Z. Li, M. Sunkara, L. Wang, and Y. Wang, Phil. Trans. Roy. Soc. A 342 (1993) 195. 
[4] W.R.L. Lambrecht, C.H. Lee, B. Segall, J.C. Angus, Z. Li, and M. Sunkara, Nature 364 (1993) 607.

[5] D.J. Fallon and L.M. Brown, Diamond Relat. Mater. 2 (1993) 1004.

[6] D.W. Kang, B.Q. Li, K.-B. Kim, H.-J. Li, in A. Feldman, Y. Tzeng, W.A. Yarbrough, M. Yoshikawa, M. Murakawa (eds.), III Int. Conf. on Applications of Diamond Films and Related Materials, NIST, Washington, 1995, p. 337

[7] G. Jungnickel, D. Porezag, Th. Frauenheim, M.I. Heggie, W.R.L. Lambrecht, B. Segall, J.C. Angus, physica status solidi (a), 154 (1996) 109.

[8] A. Hoffman, P.J.K. Paterson, and S. Prawer, Nucl. Instr. Meth. B 52 (1990) 63

[9] X.K. Zhang, J.G. Guo, Y.F. Yao, and F. Fang, Vacuum 43 (1992) 1047.

[10] J. . Vandersande and L.D. Zoltan, in Diamond Materials IV, Edited by K. V. Ravi, J. P. Dismukes, Proc. Electrochem. Soc. Symposiums, Vol. 95-4, Pennington, 1995, p. 490.

[11] M.N. Gardos and K.V. Ravi, in Diamond Materials IV, Edited by K.V. Ravi, J.P. Dismukes, Proc. Electrochem. Soc. Symposiums, Vol. 95-4, Pennington, 1995, p. 415.

[12] A.Y. Alexensky, M.L. Baidakova, M.E. Boiko, V.Y. Davydov, and A.Y. Vul, in Applications of Diamond Films and Rel. Mater: Third Intern. Conf., Edited by A. Feldman, Y. Tzeng, W.A. Yarbrough, M. Yoshikawa, M. Murakawa, NIST Publ. 885, Washington, 1995, p. 457.

[13] Z. Li, L. Wang, T. Suzuki, A. Argoitia, P. Pirouz, and J.C. Angus, J. Appl. Phys. 73 (1993) 711

[14] M.I. Heggie, G. Jungnickel, and C.D. Latham, Diamond Relat. Mater., 5 (1995) 236.

[15] M. Frenklach and H. Wang, Phys. Rev. B 43 (1991) 1520

[16] W.A. Yarbrough and R. Messier, Science 247 (1990) 688

[17] C.D. Latham, M.I. Heggie, and R. Jones, Diamond Relat. Mater. 2 (1993) 1493; C.D. Latham, M.I. Heggie, R. Jones, and P.R. Briddon, Diamond Relat. Mater. 3 (1994) 1370.
[18] Y. Matsui and H. Yabe, Diamond Relat. Mater. 2 (1993) 7.

[19] S.J. Harris, Appl. Phys. Lett. 56 (1990) 2298.

[20] S. Skokov, B. Weiner, and M. Frenklach, J. Phys. Chem. 98 (1994) 7073.

[21] S. Skokov, B. Weiner, and M. Frenklach, J. Phys. Chem. 99 (1995) 5616.

[22] B.N. Davidson and W. Pickett, Phys. Rev. B 49 (1994) 14770.

[23] G. Jungnickel, Th. Frauenheim, D. Porezag, W.R.L. Lambrecht, B. Segall, and J.C. Angus, in Mechanical Behaviour of Diamond and Other Forms of Carbon, Edited by M. Drory, D. Bogy, M. Donley, and J. Field, Proceedings Mater. Res. Soc. Symp., in press, Pittsburgh, 1995.

[24] M.I. Heggie, C.D. Latham, B. Jones, and P.R. Briddon, in Diamond Materials IV, Edited by K.V. Ravi, J.P. Dismukes, Proc. Electrochem. Soc. Symp., Vol. 95-4, Pennington, 1995, p. 643.

[25] A. De Vita, G. Galli, R. Car, and A. Canning, Bull. Am. Phys. Soc. 40 (1985) 597; A. De Vita, G. Galli, A. Canning, and R. Car, "Surface induced diamond to graphite transition probed by first principles simulations", preprint.

[26] D. Porezag, Th. Frauenheim, Th. Köhler, R. Kaschner, G. Seifert, Phys. Rev. B 51 (1995) 12947.

[27] W. Kohn and L.J. Sham, Phys. Rev. 140 (1990) A1135.

[28] R. Jones, Molecular Simulation 4 (1989) 113.

[29] R. Jones and A. Sayyash, J. Phys. C, Solid St. Phys. 19 (1986) 653.

[30] R. Jones, J. Phys. C, Solid St. Phys. 21 (1988) 5735.

[31] D.M. Ceperley and B.J. Alder, Phys. Rev. Lett. 45 (1980) 566.

[32] J.P. Perdew and A. Zunger, Phys. Rev. B 23 (1981) 5048.

[33] G.B. Bachelet, D.R. Haaman, and M. Schlüter, Phys. Rev. B 26 (1982) 4199.

[34] Th. Frauenheim, P. Blaudeck, U. Stephan, and G. Jungnickel, Phys. Rev. B 48 (1993) 4823.

[35] U. Stephan, Th. Frauenheim, P. Blaudeck, G. Jungnickel, Phys. Rev. B 49 (1994) 1489. 